\title{
Global phosphoproteomic analysis identifies SRMS-regulated secondary signaling intermediates
}

\author{
Raghuveera Kumar Goel ${ }^{1}$, Mona Meyer ${ }^{2}$, Marta Paczkowska² , Jüri Reimand ${ }^{2,3}$, Frederick Vizeacoumar ${ }^{4}$, \\ Franco Vizeacoumar ${ }^{4,5}$, TuKiet T. Lam ${ }^{6}$ and Kiven Erique Lukong ${ }^{1 *}$
}

\begin{abstract}
Background: The non-receptor tyrosine kinase, SRMS (Src-related kinase lacking C-terminal regulatory tyrosine and $\mathrm{N}$-terminal myristoylation sites) is a member of the BRK family kinases (BFKs) which represents an evolutionarily conserved relative of the Src family kinases (SFKs). Tyrosine kinases are known to regulate a number of cellular processes and pathways via phosphorylating substrate proteins directly and/or by partaking in signaling cross-talks leading to the indirect modulation of various signaling intermediates. In a previous study, we profiled the tyrosine-phosphoproteome of SRMS and identified multiple candidate substrates of the kinase. The broader cellular signaling intermediates of SRMS are unknown.
\end{abstract}

Methods: In order to uncover the broader SRMS-regulated phosphoproteome and identify the SRMS-regulated indirect signaling intermediates, we performed label-free global phosphoproteomics analysis on cells expressing wild-type SRMS. Using computational database searching and bioinformatics analyses we characterized the dataset.

Results: Our analyses identified 60 hyperphosphorylated (phosphoserine/phosphothreonine) proteins mapped from 140 hyperphosphorylated peptides. Bioinfomatics analyses identified a number of significantly enriched biological and cellular processes among which DNA repair pathways were found to be upregulated while apoptotic pathways were found to be downregulated. Analyses of motifs derived from the upregulated phosphosites identified Casein kinase 2 alpha (CK2a) as one of the major potential kinases contributing to the SRMS-dependent indirect regulation of signaling intermediates.

Conclusions: Overall, our phosphoproteomics analyses identified serine/threonine phosphorylation dynamics as important secondary events of the SRMS-regulated phosphoproteome with implications in the regulation of cellular and biological processes.

Keywords: SRMS, PTK70, Src, BRK, FRK, PTK6, PTK5, Non-receptor tyrosine kinase, Phosphoproteomics, Mass spectrometry

\section{Background}

SRMS also known as PTK70 belongs to the BRK family kinases whose other two members include Breast tumor kinase (BRK/PTK6) and Fyn-related kinase (FRK/PTK5) [1-4]. Like members of the Src family kinases as well as BRK and FRK, SRMS possesses the intermolecular-binding Src-homology 3 (SH3) and Src-homology 2 (SH2) domains and a catalytic kinase domain [5]. We previously

\footnotetext{
* Correspondence: kiven.lukong@usask.ca

${ }^{1}$ Department of Biochemistry, College of Medicine, University of

Saskatchewan, 107 Wiggins Road, Saskatoon, SK S7N 5E5, Canada

Full list of author information is available at the end of the article
}

characterized the enzymatic activity of SRMS and found that the unique 50 amino acid-long N-terminal region in SRMS regulates its enzymatic activity [5]. We further characterized Dok1 as the first substrate of SRMS [5]. Various cellular substrates and signaling intermediates have been reported for BRK and FRK which have aided the understanding of the pleiotropic roles played by these kinases $[1,2,6]$. However, unlike BRK and FRK, the cellular roles of SRMS are not well investigated. Previous studies have reported Dok1 and BRK as SRMS substrates [5, 7]. More recently, we profiled the tyrosine phosphoproteome of SRMS-expressing cells via

(c) The Author(s). 2018 Open Access This article is distributed under the terms of the Creative Commons Attribution 4.0 International License (http://creativecommons.org/licenses/by/4.0/), which permits unrestricted use, distribution, and reproduction in any medium, provided you give appropriate credit to the original author(s) and the source, provide a link to the Creative Commons license, and indicate if changes were made. The Creative Commons Public Domain Dedication waiver (http://creativecommons.org/publicdomain/zero/1.0/) applies to the data made available in this article, unless otherwise stated. 
quantitative mass spectrometry analysis and identified several candidate substrates of the kinase [8]. We also validated Vimentin and Sam68 as novel substrates of SRMS [8]. Functional gene enrichment analysis of the candidate SRMS substrates revealed that these proteins were implicated in various cellular processes such as cell growth, RNA processing and protein ubiquitination, among others [8].

Kinase-substrate interactions are known to modulate the formation of secondary and tertiary protein complexes in cells [9-11]. Such protein complexes are implicated in the regulation of other kinases thereby potentiating a concerted mode of signaling, leading to the modulation of various cellular and biological functions [12, 13]. Importantly, serine/threonine kinases constitute the majority of the mammalian kinome and evidence suggests that serine/ threonine and tyrosine kinases are interconnected by several protein-protein interactions and cross-phosphorylation events, indicative of global signaling cross-talks [14-16]. This inevitably suggests that a tyrosine kinase, for instance, may likely have an impact on the greater cellular phosphoproteome, extending beyond the direct phosphorylation of its substrates. The identification of such secondary or effector phosphorylation events on signaling intermediaries would be essential towards understanding the broader mechanisms of action of kinases.

Mass spectrometry has emerged as a powerful technique to identify and quantify proteins as well as associated post-translational modifications (PTMs) in almost any biological cell/tissue type [17, 18]. Label-free quantitation techniques offer a straightforward and cost-effective approach to reliably quantify peptide and proteins abundances in cells [19-21]. The technique has gained popularity in recent years and successfully applied to several phosphoproteomics studies [22-25].

In the present study, using label-free quantitation, we investigated the SRMS-regulated global phosphoproteome to identify potential secondary phosphorylation events involving phosphoserine and phosphothreonine sites. We used the $\mathrm{TiO}_{2}$-based phosphopeptide enrichment strategy as a tool to preferentially identify serine/threonine phosphorylation events in cells expressing wild-type SRMS. Our analyses identified multiple significantly upregulated phosphosites in the SRMS phosphoproteome. Using bioinformatics analyses, the upregulated proteins and phosphosites were mapped to various cellular and biological processes and cognate serine/ threonine kinases, respectively. Overall, our findings provide important insights on the cross-talks between SRMS and serine/threonine kinases in the orchestrated modulation of SRMS-regulated cellular functions.

\section{Methods}

\section{Cell culture, plasmids and transfection}

Human embryonic kidney (HEK) 293 cells were cultured at $37{ }^{\circ} \mathrm{C}$ in DMEM high glucose media (SH30243.01,
Hyclone) supplemented with 10\% FBS. The plasmid encoding GFP-SRMS wild-type has been previously described [5]. All transfections were performed on cells cultured to $70-80 \%$ confluency in $10 \mathrm{~cm}$ dishes. Briefly, $10 \mu \mathrm{g}$ of the appropriate plasmid was first added to $430 \mu \mathrm{L}$ of $0.15 \mathrm{M} \mathrm{NaCl}$ and gently vortexed for $10 \mathrm{~s}$ to mix. Next, $60 \mu \mathrm{L}$ of $1 \%$ PEI (Polyethyleneimine, cat. \#23966, Polysciences Inc.) was added to the DNA mix and vortexed briefly again. This mix was incubated at room temperature for $10 \mathrm{mins}$ and thereafter dispensed dropwise throughout the culture dish. The culture dishes were swirled to allow even distribution of the DNA-PEI mix and incubated at $37{ }^{\circ} \mathrm{C}$ overnight. The following day (24-h post-transfection) transfection efficiencies were verified by fluorescence microscopy.

\section{Antibodies}

Antibodies against GFP (sc-101,525) and SRMS (sc-374,324) were procured from Santa Cruz Biotechnologies (SCBT, USA). Phosphotyrosine antibodies, 4 G10 (\#05-321) were purchased from EMD-Millipore (EMD-Millipore, USA). Secondary goat anti-mouse antibodies (IR Dye-800CW IgG, \#926-32,210) were purchased from Li-Cor Odyssey, USA.

\section{Protein digestion and peptide purification}

Cells expressing the GFP-tagged SRMS variants were verified under a fluorescent microscope to ensure that equivalent and over $80-90 \%$ transfection efficiencies were achieved. The transfected cells were trypsinized, washed with $1 \mathrm{X}$ PBS and counted. $3 \times 10^{6}$ cells, from each condition, were lysed in RIPA buffer containing protease and phosphatase inhibitor cocktails (Pierce ${ }^{\mathrm{rw}}$, USA). For complete lysis, the cells were sonicated using three bursts of $10 \%$ amplitude followed by two bursts of $15 \%$ amplitude for $10 \mathrm{~s}$ each. The lysates were centrifuged at 12,000 r.c.f for 10 mins and the clarified lysates collected. Total proteins were purified by chloroform:methanol:water precipitation and the precipitated proteins resuspended in $8 \mathrm{M}$ urea $\mathrm{pH} 8.0$ containing $400 \mathrm{mM} \mathrm{ABC}$. The proteins were reduced with $10 \mathrm{mM}$ DTT and alkylated with $40 \mathrm{mM}$ iodoacetamide followed by digestion at $37^{\circ} \mathrm{C}$ with Lys-C (1:100 enzyme to protein ratio) for $6 \mathrm{~h}$ and trypsin (1:100 enzyme to protein ratio) overnight. The digestion reactions were quenched with $0.1 \%$ formic acid and desalted using $\mathrm{C}_{18}$ MacroSpin columns (The Nest Group, USA). The desalted samples were dried in a speedvac and dissolved in a buffer comprising $3.5 \%$ formic acid and $0.1 \%$ trifluoroacetic acid (TFA).

\section{Phosphopeptide enrichment}

The samples were subjected to $\mathrm{TiO}_{2}$-based phosphopeptide enrichment using TopTip MicroSpin columns (Cat. \#TT1TIO, Glygen Corp., USA), as described previously $[26,27]$. Briefly, the samples were first acidified with 
$0.5 \%$ TFA and 50\% acetonitrile and loaded onto the TopTip columns. The flowthrough from each sample was collected, dried in a speedvac and resuspended in $0.1 \%$ TFA for LC-MS analysis. The columns were then washed first with $100 \%$ acetonitrile, then with $0.2 \mathrm{M}$ Sodium phosphate $\mathrm{pH} 7.0,0.5 \%$ TFA, and finally with $50 \%$ acetonitrile. The enriched phosphopeptides were eluted with $28 \%$ Ammonium hydroxide, dried in a speedvac and resuspended in $0.1 \%$ TFA. Peptide concentrations in the phosphopeptide-enriched and flowthrough samples were estimated from $\mathrm{A}_{280}$ absorbance using the NanoDrop 2000 instrument (ThermoFisher Scientific, USA). The peptide concentrations were adjusted to $0.05 \mu \mathrm{g} / \mu \mathrm{L}$ with $0.1 \%$ TFA. A 1:10 dilution of Pierce Retention Time Calibration Mixture (Cat. \#88321, ThermoFisher Scientific, USA) was further added to each sample prior to LC-MS/MS analyses.

\section{Mass spectrometry analyses}

All samples were analysed on the Q-Exactive Plus mass spectrometer (ThermoFisher Scientific) connected to a waters nanoACQUITY UPLC system equipped with a Waters Symmetry ${ }^{\circ} \mathrm{C} 18180 \mu \mathrm{M} \times 20 \mathrm{~mm}$ trap column and a $1.7 \mu \mathrm{m}, 75 \mu \mathrm{m} \times 250 \mathrm{~mm}$ nanoACQUITY UPLC column. A total of three technical replicates for each sample were analysed by LC-MS/MS. $5 \mu \mathrm{L}$ of each replicate sample $(0.05 \mu \mathrm{g} / \mu \mathrm{L})$ was injected in a randomized order with control samples interspersed throughout to allow for correction due to potential batch effects. Peptide trapping was carried out for $3 \mathrm{~min}$ at $5 \mu \mathrm{L} / \mathrm{min}$ in $99 \%$ Buffer A (0.1\% formic acid in water) and $1 \%$ Buffer B [ $(0.075 \%$ formic acid in acetonitrile] prior to eluting with linear gradients that reached $30 \%$ Buffer B at $140 \mathrm{~min}, 40 \%$ Buffer B at $155 \mathrm{~min}$, and 85\% Buffer B at $160 \mathrm{~min}$. Two blanks (1st blank comprising 100\% acetonitrile and 2nd blank comprising Buffer A) followed each injection to ensure against sample carry over. The mass spectrometer was operated in the Data-Dependent mode with the Orbitrap operating at 60,000 FWHM and 17,500 FWHM for MS and MS/MS, respectively. Full scans were acquired at a resolution of 60,000 FWHM with a maximum injection time of $120 \mathrm{~ms}$ in the Orbitrap analyzer. The fifteen most abundant ions, with charge states $\geq 2$, were selected for fragmentation by HCD (MS/MS) using normalized collision energy set to 28 and analyzed at a resolution of 17,500 FWHM with a maximum injection time of $60 \mathrm{~ms}$.

\section{Data processing and analyses}

Label-free quantitation of the raw LC-MS/MS data was performed using the Progenesis QI software (Version 3.0, Nonlinear Dynamics), as described before [27, 28]. All MS/MS spectra were searched against the SWISSProt database (taxonomy restricted to Homo sapiens, database version dated November 2015) using the Mascot search engine (Version 2.0). Data from the $\mathrm{TiO}_{2}$-enriched and flow through fractions were analyzed separately, and the resulting quantitative analyses were combined for the respective experimental conditions. Chromatographic/ spectral alignment, mass spectral peak-picking, data filtering and statistical analyses for the protein and peptide quantitation was performed on Progenesis QI. Raw mass spectral features were aligned based on their retention time using a randomly selected reference run. All other runs were automatically aligned to the reference run to minimize retention time variability between the runs. Only spectra with ion signals quantified at or above three times the standard deviation of the noise, were selected for subsequent analyses. A normalization factor for each run was calculated to account for the variability in sample loading and ionization. This normalization factor was determined by calculating the quantitative ratio of the reference run to the run being normalized, with the assumption that most proteins/peptides are not changing in the experiment. The experimental set-up grouped replicates of each condition for comparative analyses. The algorithm then calculated and tabulated raw and normalized abundances and ANOVA $p$-values (calculated as the mean difference and variance associated with the replicate data in both experimental conditions) for each quantified peptide in the dataset. The MS/MS spectra was exported as a .mgf file (Mascot generic files) for database searching. The Mascot search algorithm was used for database searching. Carbamidomethylation (Cysteine), phosphorylation (Serine, Threonine, Tyrosine), deamidation (Asparagine, Glutamine), acetylation (Lysine) and oxidation (Methionine) were specified as variable modifications. Two missed tryptic cleavages were allowed. The precursor mass tolerance was set to $10 \mathrm{ppm}$ and the fragment mass tolerance was set to $0.2 \mathrm{Da}$. Stringent conditions were set in Mascot to filter out low scoring peptides by imposing a confidence probability score $(p)$ of $<0.05$. Peptide and protein identifications were filtered at 1\% FDR using the target-decoy strategy [29]. Only phosphopeptides mapping to proteins with at least 2 tryptic peptides were considered for analyses. The Mascot search results was exported as .xml files and then imported into the processed dataset in Progenesis QI software where the Mascot-generated peptide identifications were assigned to the corresponding quantified features. Scatterplots illustrating Pearson's correlation coefficients and fold-change phosphopeptide abundance were generated in $\mathrm{R}$ using in-house scripts.

\section{Motif enrichment analyses}

Phosphopeptide sequences of 13 amino acids in length that were significantly altered between the experimental conditions ( $p$-value $=0.05$ and 3-fold upregulation) were searched for consensus sequences using the Motif-x [30] 
and PHOSIDA [31] algorithms. On Motif- $x$, the minimum number of occurrences for a motif in the dataset and the required motif significance was set to 20 and 10E-06, respectively. On PHOSIDA, the minimum score for phospho-Ser/Thr/Tyr was specified as 10 and the minimum proportion of matching sites was set to $5 \%$.

\section{IPA functional enrichment analysis}

Genes mapped from significantly upregulated phosphopeptides were used for identifying cellular and molecular processes, pathways and upstream regulators using the Ingenuity pathway analysis (IPA) software (QIAGEN Redwood City, http://www.qiagen.com/ingenuity). Genes were queried against the Ingenuity knowledge database as the reference set. The analysis was restricted to the documentation of experimentally observed findings on human genes. The Benjamini-Hochberg $(\mathrm{B}-\mathrm{H})$ multiple testing correction $(\mathrm{B}-\mathrm{H}$ corrected $p$-value $<0.05)$ scoring method was used to compute the significance for the functional enrichment analysis. Upstream regulators refer to the upstream proteins that are responsible for causing changes in the phosphorylation and/or total expression levels of the queried genes/proteins in the dataset. Activation z-scores were calculated by IPA's z-score algorithm to predict the overall activation or inhibition of the identified functional cellular processes/pathways and upstream regulators. A positive $\mathrm{z}$-score $(\mathrm{z}$-score $>0$ ) implies an overall predicted activation of the process/ pathway/upstream regulator whereas a negative z-score (z-score $<0$ ) implies an overall predicted inhibition or downregulation of the pathway/process/upstream regulator. $\mathrm{z}$-scores $\geq 2$ or $\leq-2$ are considered significant by IPA for predicted activation or inhibition, respectively. Cellular processes/upstream regulators with no z-scores imply that IPA was unable to generate prediction states for these functionalities.

\section{Results}

Global analysis of the SRMS-regulated phosphoproteome

Tyrosine kinases and serine/threonine kinases are known to exhibit dynamic cross-talks leading to a concerted mode of regulation of signaling networks [14-16]. Evidence from large-scale database curation and high-throughput experimental observations indicate that such cross-talks are modulated through secondary and tertiary protein-protein interactions around primary kinase-substrate interaction and phosphorylation events [12, 14]. We therefore sought to study the SRMS-regulated serine/threonine phosphoproteome to infer cues on its contribution to the cellular signaling network potentially regulated by SRMS. To survey these global phosphoproteomic changes we performed metal-ion enrichment-based quantitative phosphoproteomics analysis on cells ectopically expressing wild-type SRMS (Fig. 1a). Cells expressing the empty vector backbone served as control for all background phosphorylation events occurring natively in HEK293 cells. We confirmed the expression of wild-type SRMS in the cells by immunoblotting the lysates with antibodies against GFP and SRMS (Fig. 1b). As expected, immunoblotting with phosphotyrosine antibodies confirmed the enzymatic activation of wild-type SRMS in these cells (Additional file 1: Figure S1). The total proteins were then subjected dual enzymatic digestion with Lys-C and trypsin followed by phosphopeptide enrichment using titanium dioxide $\left(\mathrm{TiO}_{2}\right)$ resin, prior to LC-MS/MS analyses (Fig. 1c).

The experiment was performed in three replicates to ensure statistical reproducibility of our analysis. Collectively, we identified 995 unique phosphosites from 1459 redundant phosphopeptides which mapped to 439 unique phosphoproteins, at an estimated False Discovery Rate (FDR) of 1\% (Fig. 2a, Additional file 2: Table S1). We found significant reproducibility between the replicate datasets, as reflected by a Pearson's correlation coefficient in the range of 0.84 to 0.96 (Fig. 2b). We quantified the number of phosphoserine, phosphothreonine and phosphotyrosine sites to assess the distribution of the identified phosphoproteome. Consistent with previous studies employing metal ion-based phosphopeptide enrichment techniques [32-34], we observed that the majority of the identified phosphosites were represented by phosphoserine (85.2\%) followed by phosphothreonine sites (13.3\%) (Fig. 2c). Phosphotyrosine sites represented a minor fraction $(0.76 \%)$ of the identified phosphoproteome (Fig. 2c). This was an expected observation since previous studies have reported a similar phosphotyrosine enrichment profile using metal ion enrichment chromatography despite significant induction of cellular tyrosine phosphorylation, for instance, via pervanadate treatment [34, 35]. Additionally, singly phosphorylated peptides were more strongly represented than doubly or triply phosphorylated peptides in our dataset, consistent with previous reports [33, 36, 37] (Fig. 2d).

To gain a better understanding of the SRMS-regulated phosphoproteome, we first applied a $p$-value threshold of $\leq 0.05$ to focus on phosphopeptides that displayed statistically significant differential regulation (up or downregulation) compared to control cells (Fig. 3a and Additional file 2: Table S1). We then defined significantly regulated phosphopeptides in the wild-type SRMS phosphoproteome as a measure of the differential abundance of the corresponding average pre-cursor ion intensities in the control cells. To ensure stringent analyses, phosphopeptides quantified at average pre-cursor ion intensities $\geq 3$-fold [ $\log _{2}$ (SRMS/ control) $\geq 1.584$ ] compared to corresponding average intensities in control cells were considered hyperphosphorylated or upregulated (Fig. 3a). Similarly, phosphopeptides with average intensities $\leq 0.5$-fold $\left[\log _{2}(\mathrm{SRMS} /\right.$ control) $\leq-1$; conversely equal to 2 -fold upregulation in control cells] were 
A

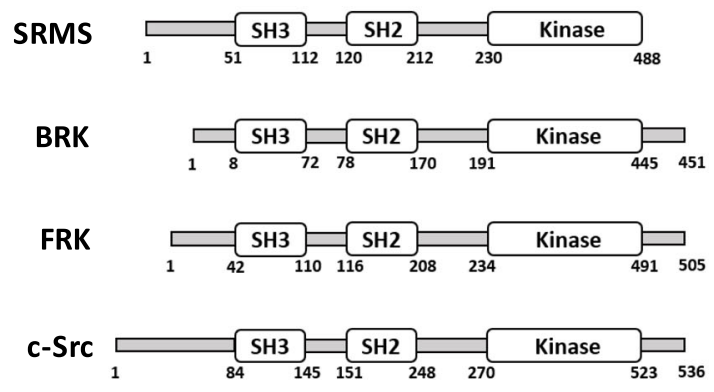

B

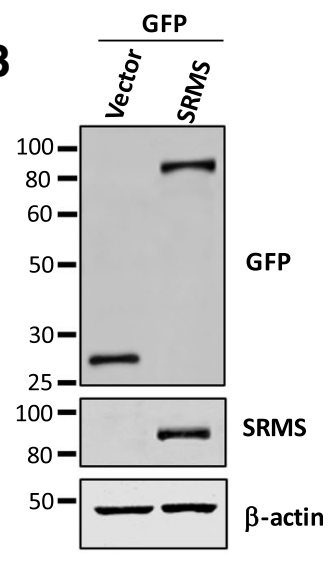

C

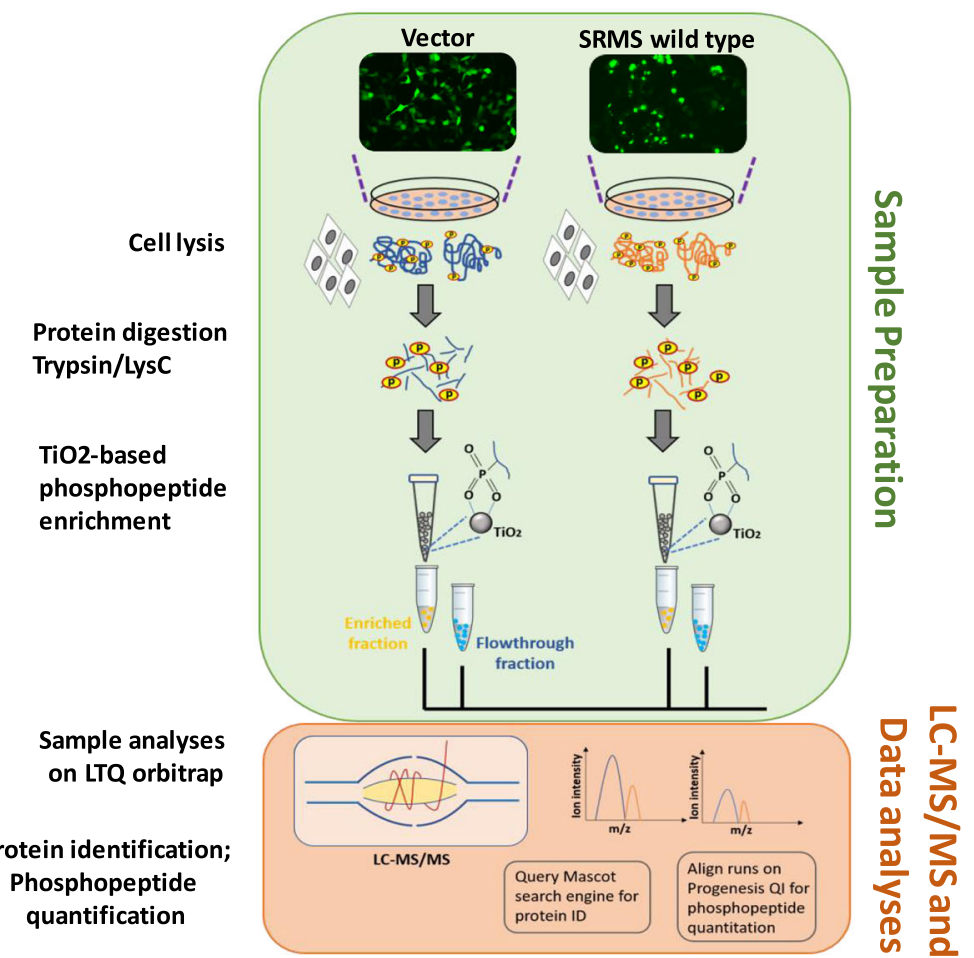

Fig. 1 Phosphoproteomics analyses of cells expressing ectopic wild-type SRMS. a Schematic representation of the domain structure of SRMS, BRK and FRK (BRK family kinases), and c-Src, depicting the SH3, SH2 and kinase domains. The amino acid numbers indicate the length of the domains and the full-length protein. $\mathbf{b}$ Immunoblotting analyses was performed on a portion of the lysates derived from HEK293 cells expressing either the empty vector (GFP alone) or vector expressing GFP-SRMS (wild-type) and used for subsequent phosphopeptide enrichment analysis. The lysates were probed with antibodies against GFP and SRMS. Immunoblotting with antibodies against $\beta$-actin was used to assess the loading of total proteins. c Schematic representation of the label-free quantitation-based phosphoproteomics workflow using cells expressing GFP alone (the empty vector control) or cells expressing GFP-SRMS wild type. The cells were lysed in RIPA buffer followed by dual enzymatic digestion (Trypsin/ Lys-C) and phosphopeptide enrichment using $\mathrm{TiO}_{2}$ resin. Both, enriched and flowthrough fractions were analysed by LC-MS/MS and data analyses performed using the MASCOT search engine (for protein identification) and PROGENESIS QI tool (for phosphopeptide quantitation)

considered hypophosphorylated or downregulated (Fig. 3a). Using these criteria, we identified 140 upregulated and 1 downregulated (NUCKS S19) phosphopeptides in the wild-type SRMS-regulated phosphoproteome (Fig. 3a and Additional file 2: Table S1). Some of the significantly upregulated phosphosites included NUCL S206, NUCL S184, MYH9 S1943, HS90A S263 and MARCS
S147. Overall, these phosphopeptides mapped to 60 upregulated and 1 downregulated proteins, respectively (Table 1 and Additional file 2: Table S1). (Table 1 is appended after the "Discussions" section in the present manuscript). We used the 0.5 -fold cut-off for downregulation since our dataset comprised only 37 phosphopeptides below the median 1-fold differential abundance ( $p$-value 


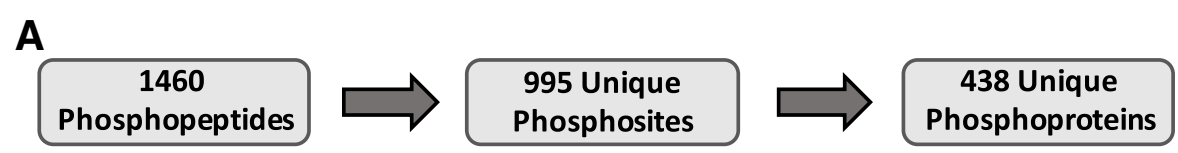

B

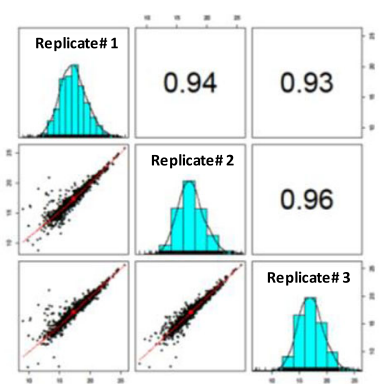

Vector

\section{Distribution of phosphorylated serine, threonine and tyrosine residues}

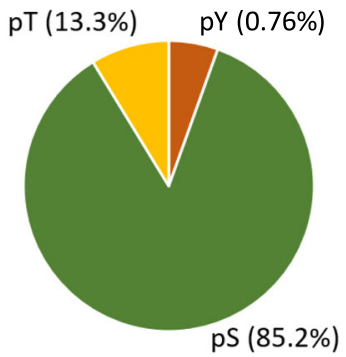

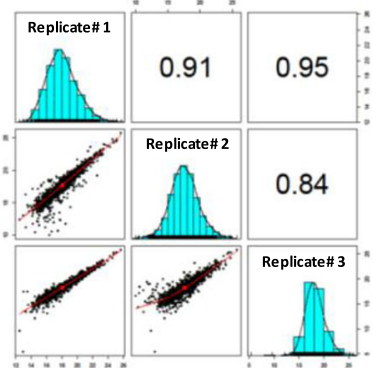

Wild type SRMS

\section{Multiplicity of phosphorylation}

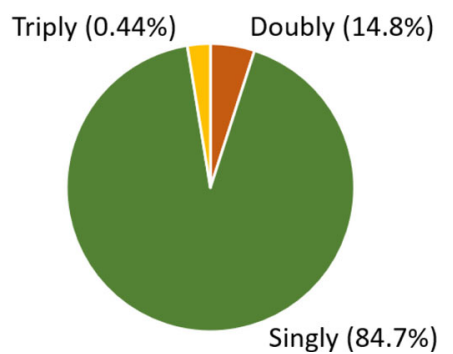

Fig. 2 Identification of the phosphoproteome. a The coverage of the phosphoproteome showing the total number of identified phosphopeptides, unique phosphosites and unique proteins mapping from the phosphopeptides. b Pearson's correlation analyses of the 3 replicate datasets corresponding to the vector control and wild type SRMS phosphoproteome. The Pearson's correlation coefficients and associated distribution curve histograms between Replicate 1, 2 and 3 for both experimental conditions are reflected in a matrix format. c Pie-chart representation of the multiplicity of phosphorylation of the identified phosphopeptides indicating the percentage of phosphopeptides carrying either a single phosphosite, double phosphosites or triple phosphosites. $\mathbf{d}$ Pie-chart depiction of the proportion of phosphoserine, phosphothreonine and phosphotyrosine sites in the identified phosphoproteome

$\leq 0.05)$ and most of these peptides displayed only marginal downregulation in phosphorylation (SRMS/Control $\sim 0.7$-fold). Additionally, we reasoned that phosphopeptides that are at least 2-fold more abundant in control than in the SRMS-regulated phosphoproteome would be likely to represent genuine SRMS-regulated hypophosphorylation events.

\section{Functional annotation of the SRMS-regulated phosphoproteome}

To better understand the cellular and biological processes mapped from the SRMS-regulated phosphoproteins, we performed functional gene enrichment analyses using the Ingenuity Pathway analyses (IPA) tool. We increased the stringency of our analyses by restricting functional annotations inferred exclusively from experimental observations. This led us to identify 30 molecular and cellular processes (Benjamini-Hochberg multiple testing $p$-value $<0.05)$ mapped from the majority of the hyperphosphorylated proteins $(48 / 60$ proteins or $80 \%)$ identified in the SRMS phosphoproteome (Fig. 3b and Additional file 3: Table S2). These cellular processes broadly represented key functional categories that included protein synthesis ( $p$-value range $1.11 \mathrm{E}-07$ to $9.87 \mathrm{E}-03$ ), cell cycle ( $p$-value range $8.49 \mathrm{E}-03$ to $1.03 \mathrm{E}-02)$, RNA post-transcriptional modification ( $p$-value range $3.49 \mathrm{E}-08$ to $1.95 \mathrm{E}-04)$, cell death and survival ( $p$-value range 6.30E-06 to $2.76 \mathrm{E}-02)$, cell growth and proliferation ( $p$-value range $1.35 \mathrm{E}-04$ to $1.29 \mathrm{E}-02$ ) and DNA replication and repair ( $p$-value $7.05 \mathrm{E}-08$ and 1.02E-04). Importantly, our analyses also led us to determine the predicted activation states of 4 molecular and cellular processes at a significant activation z-score threshold of \pm 2 . Specifically, cellular processes related to viral infection (20 proteins, $\mathrm{z}$-score $=3.8, p$-value $=5.45 \mathrm{E}-06$ ) and DNA repair (10 proteins, $\mathrm{z}$-score $=2.18, p$-value $=7.05 \mathrm{E}-08)$ were predicted with a significantly increased activation state while 
A
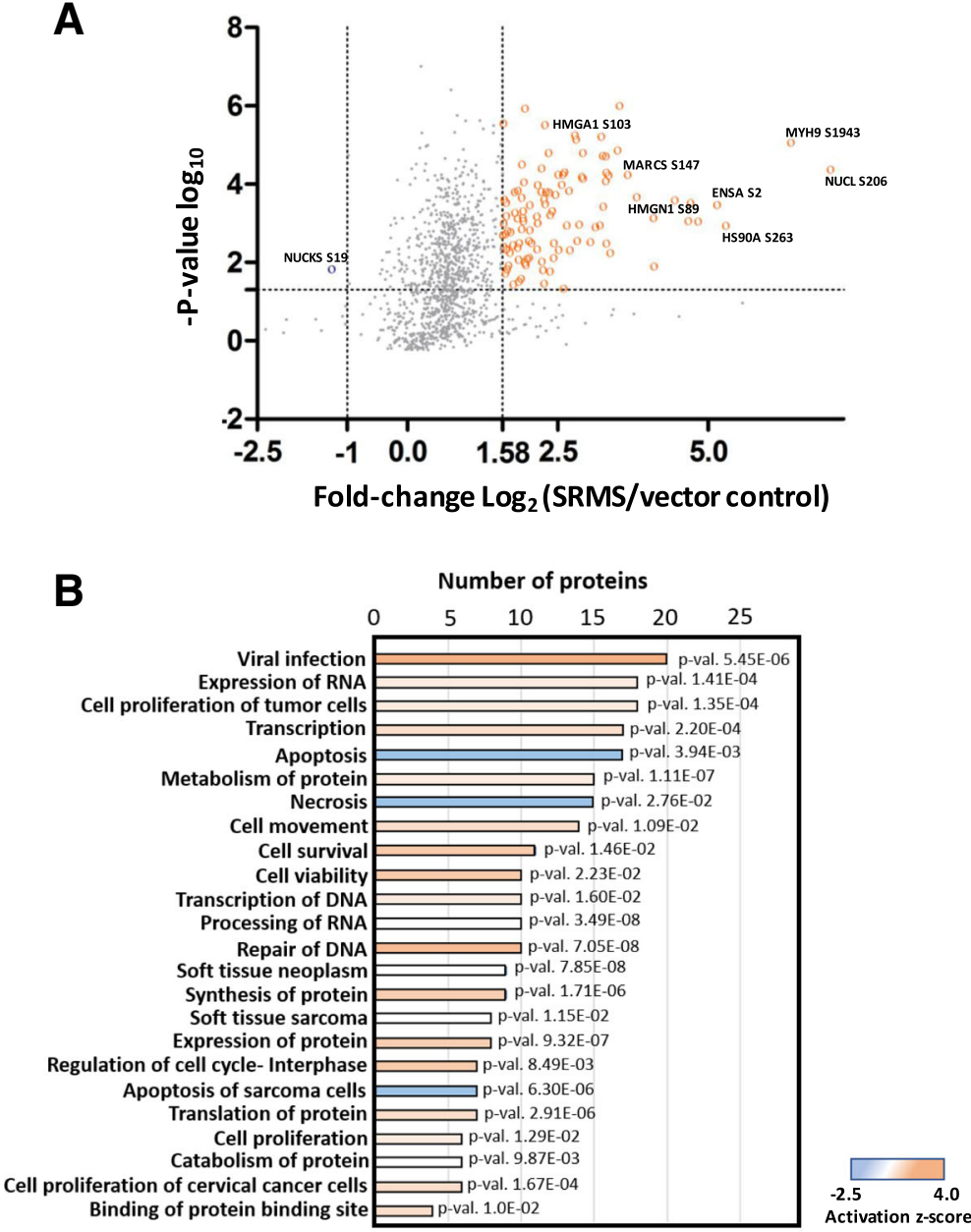

C

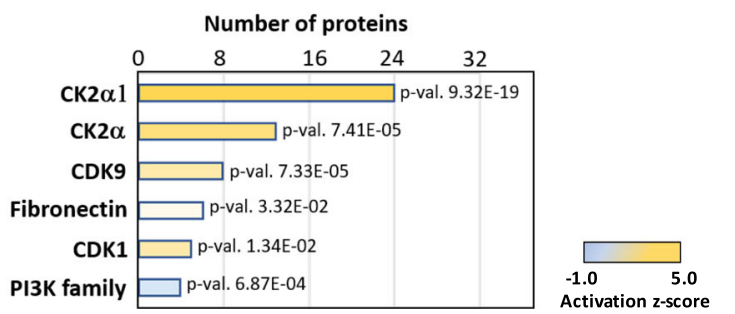

Fig. 3 Functional enrichment analyses of the significantly altered phosphoproteins. a Scatter plot showing the phosphopeptide log $_{2}$ fold-change (SRMS/control) plotted against the - $\log _{10} p$-value highlighting the significantly regulated phosphopeptides (ANOVA $p$-value $\leq 0.05$, upregulation fold-change cut-off $=\log _{2} \geq 1.58$ and downregulation fold-change cut-off $=\log _{2} \leq-1$ ). Upregulated phosphopeptides are highlighted in orange while downregulated phosphopeptides are highlighted in blue. $\mathbf{b}$ IPA analyses of the top cellular and molecular processes enriched from upregulated phosphoproteins identified in the SRMS-regulated phosphoproteome ( $n=60$; corresponding to upregulated phosphopeptides; SRMS/Control Log $2 \geq 1.58-$ fold). The activation z-score indicates the predicted upregulation (z-score $>1$ ) or downregulation (z-score $<0$ ) of specific cellular and molecular processes. $\mathbf{c}$ IPA analyses of upstream regulators for the upregulated phosphoproteins $(n=60)$. $z$-score indicates the predicted activation $(z$-score $>1)$ or inactivation $(z$-score $<0)$ of the indicated upstream proteins

apoptosis (17 proteins, $\mathrm{z}$-score $=-2.11, p$-value $=3.94 \mathrm{E}-03$ ) and necrosis (15 proteins, $z$-score $=-2.48, \quad p$-value $=$ $2.76 \mathrm{E}-02)$ were predicted to display an overall decreased activation state (Fig. $3 \mathrm{~b}$ and Additional file 3: Table S2). Some of the other interesting cellular/biological themes enriched from the hyperphosphorylated proteins included cell movement $(p$-value $=1.35 \mathrm{E}-04)$ and catabolism of protein $(p$-value $=9.87 \mathrm{E}-03)$ (Fig. $3 \mathrm{~b}$ and Additional file 3: Table S2). Analyses of the upstream regulators identified 5 proteins, namely, PI3K (Phosphoinositol-3-kinase), CDK9 (Cyclin-dependent kinase 9), CK2 (Casein kinase 2), CK2 $\alpha$ (Casein kinase 2 catalytic subunit alpha) and FN1 
Table 1 SRMS-dependent upregulated phosphoproteins

\begin{tabular}{|c|c|c|}
\hline Protein Accession (UniProt ID) & Phosphosite(s) & Protein Description \\
\hline AHNK & S5731, S5739 & Neuroblast differentiation- associated protein \\
\hline AKA12 & S248, S381, S732, S743, S749 & A-kinase anchor protein 12 \\
\hline BAD & S75, S118 & Bcl-associated agonist of cell death \\
\hline CALX & S554, S564 & Calnexin \\
\hline DKC1 & S451, S453, S455, S458, S494 & H/ACA ribonucleoprotein complex subunit 4 \\
\hline $\mathrm{FOXO3}$ & T51, S55 & Forkhead box protein $\mathrm{O} 3$ \\
\hline HS90 & S252, S263 & Heat shock protein HSP 90-alpha \\
\hline HMGA1 & S102 & High mobility group protein HMG-I/HMG-Y \\
\hline IF2P & S107, S113 & Eukaryotic translation initiation factor 5B \\
\hline MAP1B & S1016, S1154, S1869, T1879 & Microtubule-associated protein 1B \\
\hline MARCS & $\mathrm{S} 147, \mathrm{~T} 150$ & Myristoylated alanine-rich C-kinase substrate \\
\hline $\mathrm{NUCL}$ & S145, S184, S206 & Nucleolin \\
\hline NUDC & S136, S139, S145 & Nuclear migration protein nudC \\
\hline SFR19 & Y305, T335, T994, S998, T1001 & Splicing factor, argining/serine-rich 19 \\
\hline SRRM1 & S465, S775 & Serine/arginine repetitive matrix protein 1 \\
\hline SRRM2 & S144, T1413 & Serine/arginine repetitive matrix protein 2 \\
\hline TCP4 & S9, S17, S19 & Activated RNA polymerase II transcriptional coactivator p15 \\
\hline
\end{tabular}

Shown here is a representative list of selected hyperphosphorylated/upregulated proteins identified in the wild-type SRMS phosphoproteome

(Fibronectin) (Fig. 3c and Additional file 3: Table S2). Importantly, among these, 2 kinases were associated with significantly increased predicted activation- the tetrameric kinase complex, CK2 (z-score $=3.54, p$-value $=7.41 \mathrm{E}-05)$, its monomeric catalytic subunit, CK2 $\alpha$ (z-score $=4.87$, $p$-value $=9.32 \mathrm{E}-19)$, and CDK9 $(\mathrm{z}$-score $=2.81, p$-value $=$ 7.33E-05). Further, our analyses also revealed that a small number of the hyperphosphorylated proteins were enriched in specific signaling pathways (Additional file 3: Table S2) which included Telomerase signaling (-Log $p$-value $=2.33)$, PI3K/AKT signaling $(-\log p$-value $=2.18)$, Sirtuin signaling $(-\log p$-value $=1.93)$ and Phospholipase $\mathrm{C}$ signaling $(-\log p$-value $=1.44)$.

We next used the STRING database to generate a protein-interaction network of the SRMS-regulated hyperphosphorylated proteins. STRING allows for the analyses of protein-protein interactions of a given set of genes based on correlation with predicted and experimental sources [38]. Our analyses using STRING's curated database of human protein interactions resulted in a network of 38 interconnected nodes (proteins) characterized by 59 edges (connections) implying a moderate level of interaction among the hyperphosphorylated proteins (Additional file 4: Figure S2). This reasonably moderate degree of connectivity among 38 phosphoproteins (63\% of total SRMS-regulated hyperphosphorylated proteins) suggests that a sizeable number of the SRMS-regulated phosphoproteins potentially partake in the same protein complex and possibly the same cellular signaling processes.

\section{Analyses of phosphorylation motifs and predicted kinases} The amino acids surrounding a phosphosite constitute important recognition motifs for cognate kinases [39-41]. Our phosphoproteomics analysis identified 140 upregulated phosphopeptides that mapped to 60 proteins in the SRMS-regulated phosphoproteome. We assessed the enrichment of motifs among these upregulated phosphopeptides using the Motif- $x$ and PHOSIDA motif-enrichment tools [30, 31]. Our analyses using both the tools resulted in the enrichment of similar motifs ( $p$-value $=10 \mathrm{E}-06$ ) which primarily corresponded to phosphoserine sites (Fig. 4a, b and c). No significant phosphothreonine or phosphotyrosine motifs were found due to their lower abundances in our dataset. Collectively, we identified four major motifs; all comprising positional variations in amino acids following the central phosphoserine residue. One significantly overrepresented motif comprised a glutamic acid and aspartic acid residue in the +3 and +5 positions $(S x x E x D)$ (Fig. 4a, b and c). Other top motifs comprised either a glutamic acid and a serine residue at the +2 and +3 positions $(S x E S)$, a serine at +2 position $(S x S)$ or a glutamic acid residue at +3 position $(S x x E)$ (Fig. $4 \mathrm{a}, \mathrm{b}$ and c).

We further analyzed the upregulated phosphopeptides using the NetworKIN tool [42] to identify candidate kinases responsible for the site-specific phosphorylation of the associated motifs. The NetworKIN algorithm exploits information based on consensus motif recognition as well as context-specific factors such as the physical association and co-expression of kinases and substrates, to predict corresponding kinase-substrate relationships 

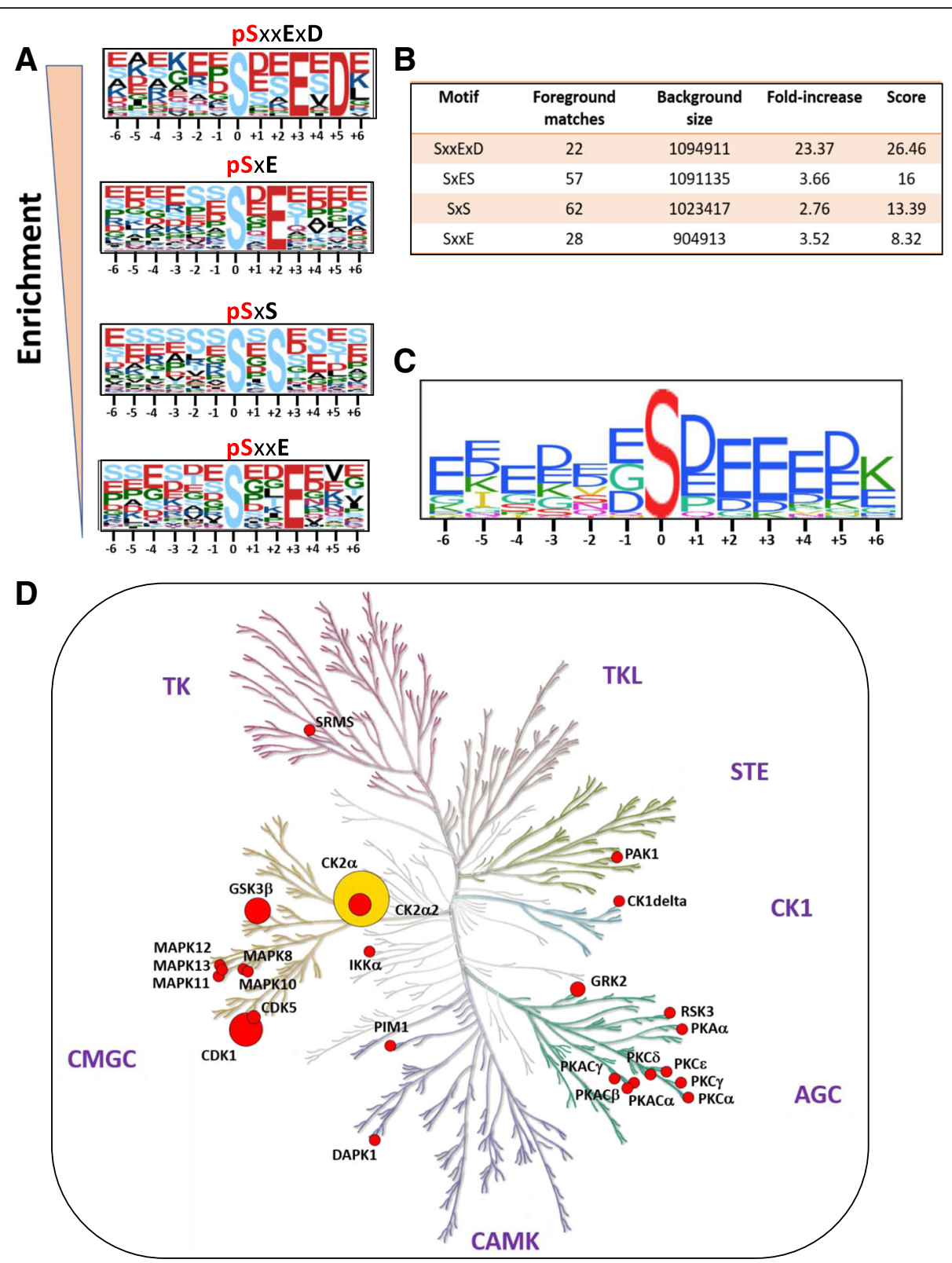

Fig. 4 Motif-enrichment analyses of SRMS-dependent upregulated phosphopeptides. a Motif-logos showing the significantly enriched motifs ( $p$-value <10E-06) identified by Motif-x [30]. The positions of the amino acid residues C-terminal or N-terminal to the central phosphoresidue (position " 0 ") are shown in the logos. The height of the amino acid residues is proportional to their enrichment at the specific position in the pool of the queried phosphopeptides. $\mathbf{b}$ Table representing the scoring information relevant to the enriched motifs identified by Motif-x. $\mathbf{c}$ A consolidated motif-logo generated by PHOSIDA [31] showing the enrichment of various amino-acid residues at specific positions relative to the central phosphoresidue (position "0"). d Dendogram of the human kinome, constructed by KinMap [69], highlighting the candidate kinases predicted to target the upregulated phosphopeptides, as identified by NetworkIN [42] analysis. Node size is proportional to the number of the queried phosphosites targeted by the kinase. CK2 (node highlighted in yellow) was identified as the upstream kinase for the maximum number of queried phosphosites. Major kinase families are annotated in the dendogram which include: TK (Tyrosine Kinases), TKL (Tyrosine Kinase-Like), STE (Sterile kinases; homologs of the yeast STE7, STE11 and STE20 kinases), CK1 (Casein Kinase 1), AGC (comprising Protein kinase AV PKA, PKG and PKC kinase sub-families), CAMK (Calcium/ Calmodulin-dependent kinases) and CMGC (comprising cyclin-dependent kinase (CDK), mitogen-activated protein kinase (MAPK), glycogen synthase kinase (GSK) and CDC-like kinase (CLK))

[42, 43]. NetworKIN analyses of the hyperphosphorylated motifs identified 25 candidate serine/threonine kinases, corresponding to 16 kinase subfamilies, at a NetworKIN score $>3$ (Fig. 4d and Additional file 5: Table S3). Kinases found to potentially target multiple phosphosites included CDK1 (5 phosphosites, NetworKIN score range: 9.62-17.93), CK2 alpha (19 phosphosites, score range: 3.17-35.63), GSK3 beta (5 phosphosites, score range: 
4.75-10.43), CK2 alpha 2 (3 phosphosites, score range: 8.47-9.61), GRK2 (3 phosphosites, score range: 4.309.76) and CDK5 (2 phosphosites, score range: 3.4214.02) (Fig. $4 \mathrm{~d}$ and Additional file 5: Table S3). Importantly, Casein Kinase 2 alpha $(\mathrm{CK} 2 \alpha)$ was the among the highest scoring candidate kinases and found to target the maximum number of phosphosites in our dataset (Fig. 4d and Additional file 5: Table S3). CK2 $\alpha$ is a functionally independent catalytic subunit of the tetrameric holoenzyme, CK2 [44]. The consensus recognition motifs for the acidophilic kinase, $\mathrm{CK} 2 \alpha$, are characterized by the presence of either glutamic acid or aspartic acid residues near the phosphorylation site [43, 45]. Indeed, the overrepresentation of such motifs $(S x x E x D, S x E$ and $S x x E$ ) by motif enrichment analyses confirms CK $2 \alpha$ as a major upstream kinase of the upregulated phosphosites. This also corroborated findings from our functional gene enrichment analyses using IPA where the holoenzyme CK2 and its catalytic module, Casein kinase 2 alpha were identified as major upstream regulatory kinases of the upregulated phosphoproteins (Fig. 3c). Taken together, our findings suggest that CK2 $\alpha$ represents a key serine/threonine kinase that is potentially activated in the SRMS phosphoproteome. This further indicates that CK2a and/or CK2 may likely represent key downstream targets of SRMS.

\section{Discussion}

Here we used quantitative mass spectrometry analyses to identify cognate serine/threonine phosphorylation events significantly altered in the SRMS-regulated phosphoproteome. We identified 60 phosphoproteins mapped from 140 phosphopeptides which were upregulated by at least 3-fold in the SRMS-regulated phosphoproteome. To our knowledge, this represents the first study to investigate global serine and threonine phosphorylation changes induced by a bonafide non-receptor tyrosine kinase in the eukaryotic phosphoproteome.

Despite the ectopic expression of SRMS in cells, we identified only $1.3 \%$ phosphotyrosine peptides in contrast to our previous study where we reported multiple phosphotyrosine peptides [8] (Fig. 2d). The lysis of cells under less stringent conditions, such as by RIPA lysis buffer used in this study, and the use of $\mathrm{TiO}_{2}$ resin, known to less favorably bind to phosphotyrosines, are factors that may explain the lower enrichment of tyrosine-phosphorylated peptides. The former may be an equally important factor since tyrosine phosphorylation is far more stringently regulated by phosphatase activity than serine/threonine phosphorylation [22] and as such the use of denaturing conditions during cell lysis have proved useful in such studies [22, 46, 47]. The relatively lower selectivity of $\mathrm{TiO}_{2}$ resin towards phosphotyrosine peptides can also be demonstrated by the fact that we identified only 2 major phosphorylation sites on SRMSY299 and Y456, in this study. Our previous phosphotyrosine enrichment-based approach enabled the identification of several other phosphotyrosine sites on wild-type SRMS [8]. However, since the focus of this study was to identify phosphoserine and phosphothreonine events, the relatively poor enrichment of phosphotyrosine peptides did not pose a concern.

The cellular roles of SRMS have not been well characterized to date. In the present study, functional enrichment analyses of the upregulated phosphoproteins in the SRMS-regulated phosphoproteome identified apoptosis and necrosis as potentially important downregulated cellular processes in cells expressing ectopic SRMS. A previous study by Potts et al. implicated SRMS in the potential negative regulation of autophagy [48], implying that SRMS may potentially be a positive regulator of stress-induced cell survival. This is in line with our present findings which not only projected a downregulation of apoptotic processes but also a potential upregulation of cell survival and proliferative processes in cells expressing ectopic SRMS, as determined by a positive z-score from IPA analyses (Fig. 3b and Additional file 3: Table S2). Interestingly, processes related to DNA repair were also predicted to be upregulated (Fig. $3 \mathrm{~b}$ and Additional file 3: Table S2). As an example, HMGA1 was among the upregulated proteins enriched in DNA repair processes (Additional file 3: Table S2). We identified HMGA1 S102 as a hyperphosphorylated site in cells expressing ectopic SRMS (Additional file 2: Table S1). HMGA1 S102 is a target site for phosphorylation by CK2 $[49,50]$ and studies have shown that the hyperphosphorylation of HMGA1 S102 impairs the DNA-binding ability of the protein $[51,52]$. The dissociation of HMGA1 from the DNA in turn promotes efficient DNA repair, presumably by allowing various DNA repair factors to be recruited to the sites of DNA lesions, as reported previously [53, 54]. Taken together, our data points towards a potential role of SRMS in modulating cellular DNA repair processes by regulating HMGA1 S102 phosphorylation. Further, HMGA1 has also been shown to inhibit apoptosis by suppressing p53-mediated transcriptional repression of apoptosis-related genes like Mdm2, Bax and p21 [55]. This therefore also explains the cross-talk involving HMGA1 between cellular processes linked to DNA repair, apoptosis and ell proliferation, in our dataset (Additional file 3: Table S2).

Analyses of the phosphosites using the Motif-x, PHOSIDA and NetworKIN tools identified CK2 as one of the major candidate upstream kinase for the upregulated phosphosites identified in the SRMS-regulated phosphoproteome (Fig. 4a-d, Additional file 3: Table S2). This raises the possibility that SRMS may potentially function upstream of CK2 and as a candidate regulator of CK2 
activity. CK2 is an active tetrameric serine/threonine kinase composed of two catalytic subunits ( $\alpha$ and $\left.\alpha^{\prime}\right)$ and two regulatory $\beta$-subunits [56]. Activation of CK2 is primarily regulated in vivo by inositol phosphates $[57,58]$, phospholipase D2 (PLD2) and protein kinase C (PKC) [59]. However, previous studies have identified c-Abl, BCR-Abl [60] and Src-family kinases, Lyn and c-Fgr [61] as regulators of CK2 activity. Specifically, the catalytic subunit of CK2, CK2 $\alpha$, was identified as a substrate of these kinases where phosphorylation by c-Abl or BCR-Abl was shown to inhibit CK2 $\alpha$ activity [60] while phosphorylation by Lyn or c-Fgr was shown to increase CK2 $\alpha$ activity [61]. Interestingly, our previous phosphotyrosine enrichment-based phosphoproteomics analysis found CK $2 \alpha$ to be significantly tyrosine-phosphorylated exclusively in cells expressing ectopic wild type SRMS, projecting CK $2 \alpha$ as a candidate SRMS substrate [8]. Specifically, CK2 $\alpha$ Y182, Y188 and Y323 were identified as hyperphosphorylated sites. Of these, Y182 represents a key residue lying in the activation loop of the $\mathrm{CK} 2 \alpha$ subunit and the trans-autophosphorylation of this site has been shown to increase CK2 $\alpha$ activity [62]. Additionally, CK2 Y188 has also been reported as another activation loop phosphosite contributing to $\mathrm{CK} 2 \alpha$ activation, albeit to a lesser extent than CK2 Y182 [62, 63]. However, findings from our previous phosphoproteomics study indicate that CK2 $\alpha$ Y182/Y188 may potentially serve as target sites of SRMS leading to the SRMS-dependent modulation of CK $2 \alpha$ enzymatic activity. Overall, this supports our present findings which indicate that CK2 $\alpha$ is potentially activated in cells overexpressing wild type SRMS which may highlight SRMS as a possible modulator of CK2 $\alpha$ kinase-dependent functions.

CDK1/cdc2 is another upstream candidate kinase identified by NetworKIN analysis which was also identified as an upstream regulator by IPA analyses. Kinases Wee1 [64], Myt1 [65] and Lyn [66] are known to regulate the activity of CDK1 by phosphorylating the inhibitory Y15 site on CDK1. Our previous phosphoproteomics study also identified CDK1 as a candidate target of SRMS where CDK1 Y19, Y270 and Y286 were found to be hyperphosphorylated [8]. However, these sites on CDK1 have not been functionally characterized. Therefore, the mechanism by which CDK1 may be potentially activated in the presence of SRMS, leading to the phosphorylation of the CDK1 consensus phosphosites, is not known.

It is important to note that the overall cellular and molecular processes mapped from the SRMS-regulated signaling intermediates identified in the present study, were consistent with the major functional themes mapped from the candidate SRMS substrates in our previous study [8]. Some of the major functional themes enriched by the candidate SRMS substrates included RNA processing, Viral processes, negative regulation of apoptosis, cell cycle regulation and protein ubiquitination [8]. These correlate well with the broad functional categories identified in the present study such as RNA post-transcriptional modifications (Processing of RNA), viral infection, cell death and survival (apoptosis), cell cycle, and protein degradation, respectively (Additional file 3: Table S2). Collectively, our findings here indicate that some of the major cellular processes regulated by SRMS involve additional signaling factors characterized by serine/threonine phosphorylation events downstream of cognate serine/threonine kinases. Our study therefore presents key evidence that serine/ threonine phosphorylation forms part of important secondary signaling events triggered by SRMS. Overall, our findings provide an important mechanistic resource to characterize the cellular roles played by SRMS.

\section{Conclusions}

Our global phosphoproteomic profiling reveals that the non-receptor tyrosine kinase, SRMS can indirectly regulate multiple signaling intermediates which are characterized by an altered serine/threonine phosphorylation status. The regulation of serine/threonine phosphorylation events in the presence of SRMS is likely a result of SRMS-dependent direct or indirect modulation of serine/ threonine kinase activity. In this context, the serine/threonine kinase, Casein kinase 2 (CK2) may likely represent one of the major downstream targets of SRMS. Overall, findings from our study form an important mechanistic resource for characterizing the cellular role(s) of SRMS in mammalian cells.

\section{Additional files}

Additional file 1: Figure S1. Immunoblotting analysis with antibodies against total phosphotyrosines on lysates derived from cells expressing either empty vector control (GFP alone) or GFP-SRMS wild type. Antibodies against $\beta$-actin were used for immunoblotting to assess the loading of total proteins. (TIF $855 \mathrm{~kb}$ )

Additional file 2: Table S1. The table is organized in the following spreadsheets: Spreadsheet "All Phosphopeptides": This table lists all identified phosphopeptides from three independent replicates, via LCMS/MS. Additional information corresponding to each phosphopeptide is also shown and includes peptide ID\# (numerical ID), fraction (enriched; 1 or flowthrough; 2 fractions), Retention time, $\mathrm{m} / \mathrm{z}$, charge, measured mass, mass error, score, peptide sequence, modifications (type of PTM), UniProt accession, Phosphosite(s), sequence window, description of protein, use in quantitation (at least 2 unique tryptic peptides were identified for the corresponding protein), ANOVA p-values corresponding to each phosphopeptide identified across the three independent replicates, normalized abundance (phosphopeptide intensity) of the identified phosphopeptides across each replicate, average phosphopeptide intensities of the peptide in the control cells or cells expressing wild type SRMS, foldchange abundance of phosphopeptide intensities in SRMS-expressing cells (SRMS/Control) and Log $_{2}$-scaled fold-change of SRMS/control values. Spreadsheet "All unique phosphoproteins": This table lists all the unique phosphoproteins mapped from the phosphopeptides quantified via LC-MS/MS in the Vector and SRMS-expressing cells. Also shown are all associated phosphosites identified for each protein. Spreadsheet "Phosphopeptides $p$-value $\leq 0.05$ ": This table lists all phosphopeptides 
filtered at an ANOVA p-value threshold of 0.05 . Also shown is other relevant information corresponding to each phosphopeptide, as in Spreadsheet "All Phosphopeptides". Spreadsheet "Hyperphosphorylated proteins": This table lists all the hyperphosphorylated/upregulated proteins identified in the SRMS-regulated phosphoproteome. These proteins were mapped from the hyperphosphorylated/upregulated peptides ( $\log _{2}$ SRMS/ Control $\geq 1.58$ and ANOVA $p$-value $\leq 0.05$ ). Therefore, only phosphosites identified at $\log _{2}$ SRMS/Control $\geq 1.58$ and ANOVA $p$-value $\leq 0.05$, are shown for every protein. Spreadsheet "Hypophosphorylated proteins": Shown here is the hypophosphorylated/downregulated protein mapped from the hypophosphorylated/downregulated peptide ( $\log _{2}$ SRMS/Control $\leq-1$ and ANOVA $p$-value $\leq 0.05$ ) identified in SRMS-expressing cells. (XLSX $745 \mathrm{~kb})$

Additional file 3: Table S2. The file is organized into the following spreadsheets: Spreadsheet "Cell. \& Biol. processes": This table shows all the cellular and biological processes identified by IPA analysis (www.ingenuity.com) enriched from the upregulated/ hyperphosphorylated proteins identified in the SRMS-regulated phosphoproteome. To minimize redundancy, the cellular and biological processes are categorized in specific functional "categories". Also shown for every cellular and biological process are $P$-values (Fisher's exact t-test), activation $z$-score, predicted activation state (based on a cut-off $z$-score $=+/-$ 2), protein names and the number of proteins enriched in the corresponding cellular and biological process. Spreadsheet "Upstream regulators": This table lists all the upstream regulators identified by IPA analysis for the SRMS-dependent upregulated/hyperphosphorylated proteins. Also shown are $p$-values, activation $z$-score, predicted activation state (based on a cut-off $z$-score $=+/-2$ ), protein names and number of proteins for every upstream regulator identified by IPA analyses. Spreadsheet "Signaling pathways": Shown here are the canonical signaling pathways corresponding to the upregulated/hyperphosphorylated proteins, as identified by IPA analysis. Also shown are the - $\log p$-values, ratio (the ratio estimates the representation of the proteins in each canonical pathway. This is calculated as the ratio of the number of proteins that are enriched in the pathway to the number of proteins in the Ingenuity database reference dataset that make up the pathway) and the protein names corresponding to each canonical pathway. (XLSX $15 \mathrm{~kb}$ )

Additional file 4: Figure S2. A protein-protein interaction network map constructed using STRING [38] showing the intermolecular associations between the upregulated phosphoproteins (represented by nodes) at an interaction score threshold set to 0.4 (medium confidence). Nodes interconnected by an edge are representative of a protein-protein interaction context, as determined by experimental observations (edges highlighted in Pink), database searches (edges highlighted in blue) and text-mining (edges highlighted in green). (TIF $5774 \mathrm{~kb}$ )

Additional file 5: Table S3. This table lists all the candidate upstream kinases identified by NetworkIN analysis, for the upregulated phosphosites. Shown in the table is the substrate (phosphoprotein identified in the SRMS-regulated phosphoproteome), position (corresponding upregulated phosphosite), upstream kinase ID (candidate kinase known to target the corresponding phosphosite), NetworkIN score, kinase group (NetPhorest-annotated kinase group), NetPhorest score, STRING identifier (the corresponding STRING-annotated identifier/D for the interaction between the kinase and its substrate phosphoprotein), STRING score, Motif sequence (the specific motif corresponding to the phosphosite that is targeted by the candidate kinase) and STRING path. (XLSX $16 \mathrm{~kb})$

\footnotetext{
Abbreviations

BFK: BRK family kinase; BRK: Breast tumor kinase; Cdc2: Cell division cycle protein 2; CDK1: Cyclin-dependent kinase 1; CDK9: Cyclin-dependent kinase 9; CK2: Casein kinase 2; CK2a: Casein kinase 2 alpha; FDR: False discovery rate; FRK: Fyn-related kinase; HMGA1: High mobility group protein A1; MYH9: Myosin heavy chain 9; NUCL: Nucleolin; PI3K: Phosphoinositol 3 kinase; PTB: Phosphotyrosine-binding domain; PTK70: Protein tyrosine kinase 70; SFK: Src family kinases (SFKs); SH2: Src-homology domain 2; SH3: Srchomology domain 3; SRMS: Src-related kinase lacking C-terminal regulatory tyrosine and $\mathrm{N}$-terminal myristoylation sites
}

\section{Acknowledgements}

We would like to thank Dr. William (Bill) Roesler (Head, Dept. of Biochemistry, University of Saskatchewan) for his invaluable support.

\section{Funding}

This work was supported by the President's NSERC grant administered by the University of Saskatchewan and the NSERC Discovery grant (RGPIN-201705564).

\section{Availability of data and materials}

The mass spectrometry proteomics data have been deposited to the ProteomeXchange consortium [67] via the PRIDE partner repository [68] with the dataset identifier PXD009234. The data can be accessed on https:// www.ebi.ac.uk/pride/archive/login using the following reviewer account details: Username: reviewer12016@ebi.ac.uk

Password: HKaGSifi.

\section{Authors' contributions}

RKG is the primary and major contributor to the project. RKG and KEL conceived and designed the research. RKG, KEL, TTL, JR, MM, MP, FSV and FJV contributed to data analyses. The manuscript was written and edited by RKG and KEL. The manusript was revised by RKG, TTL, JR and KEL. All authors read and approved the final manuscript.

\section{Ethics approval and consent to participate}

Not applicable

\section{Consent for publication}

Not applicable

\section{Competing interests}

The authors declare that they have no competing interests.

\section{Publisher's Note}

Springer Nature remains neutral with regard to jurisdictional claims in published maps and institutional affiliations.

\section{Author details}

'Department of Biochemistry, College of Medicine, University of Saskatchewan, 107 Wiggins Road, Saskatoon, SK S7N 5E5, Canada.

${ }^{2}$ Computational Biology Program, Ontario Institute for Cancer Research, 661 University Ave Suite 510, Toronto, ON M5G 0A3, Canada. ${ }^{3}$ Department of Medical Biophysics, University of Toronto, 101 College Street Suite 15-701, Toronto, ON M5G 1L7, Canada. ${ }^{4}$ Department of Pathology, Cancer Cluster, College of Medicine, University of Saskatchewan, Saskatoon, SK S7N 5E5, Canada. ${ }^{5}$ Saskatchewan Cancer Agency, University of Saskatchewan, 107 Wiggins Road, Saskatoon, SK S7N 5E5, Canada. ${ }^{6}$ Department of Molecular Biophysics and Biochemistry and MS \& Proteomics Resource, WM Keck Foundation Biotechnology Resource Laboratory, Yale University, New Haven, $C T$, USA.

Received: 24 March 2018 Accepted: 19 July 2018

Published online: 18 August 2018

\section{References}

1. Goel RK, Lukong KE. Tracing the footprints of the breast cancer oncogene BRK - past till present. Biochim Biophys Acta. 2015;1856(1):39-54.

2. Goel RK, Lukong KE. Understanding the cellular roles of Fyn-related kinase (FRK): implications in cancer biology. Cancer Metastasis Rev. 2016;35(2):179-99.

3. Kohmura N, Yagi T, Tomooka Y, Oyanagi M, Kominami R, Takeda N, Chiba J, Ikawa Y, Aizawa S. A novel nonreceptor tyrosine kinase, Srm: cloning and targeted disruption. Mol Cell Biol. 1994;14(10):6915-25.

4. Kawachi $Y$, Nakauchi $H$, Otsuka F. Isolation of a cDNA encoding a tyrosine kinase expressed in murine skin. Exp Dermatol. 1997;6(3):140-6.

5. Goel RK, Miah S, Black K, Kalra N, Dai C, Lukong KE. The unique N-terminal region of SRMS regulates enzymatic activity and phosphorylation of its novel substrate docking protein 1. FEBS J. 2013;280(18):4539-59.

6. Brauer PM, Tyner AL. Building a better understanding of the intracellular tyrosine kinase PTK6 - BRK by BRK. Biochim Biophys Acta. 2010;1806(1):66-73. 
7. Fan G, Aleem S, Yang M, Miller WT, Tonks NK. Protein-tyrosine phosphatase and kinase specificity in regulation of SRC and breast tumor kinase. J Biol Chem. 2015;290(26):15934-47.

8. Goel RK, Paczkowska M, Reimand J, Napper S, Lukong KE. Phosphoproteomics analysis identifies novel candidate substrates of the non-receptor tyrosine kinase, SRMS. Mol Cell Proteomics 2018;17(5):925-47.

9. Krapivinsky G, Medina I, Krapivinsky L, Gapon S, Clapham DE. SynGAPMUPP1-CaMKII synaptic complexes regulate p38 MAP kinase activity and NMDA receptor-dependent synaptic AMPA receptor potentiation. Neuron. 2004;43(4):563-74.

10. Wegener E, Krappmann D. Dynamic protein complexes regulate NF-kappaB signaling. Handb Exp Pharmacol. 2008;186:237-59.

11. Kapoor P, Bao Y, Xiao J, Luo J, Shen J, Persinger J, Peng G, Ranish J, Bartholomew B, Shen X. Regulation of Mec1 kinase activity by the SWI/SNF chromatin remodeling complex. Genes Dev. 2015:29(6):591-602.

12. Ochoa D, Jonikas M, Lawrence RT, El Debs B, Selkrig J, Typas A, Villen J, Santos SD, Beltrao P. An atlas of human kinase regulation. Mol Syst Biol. 2016;12(12):888

13. Besset V, Scott RP, Ibanez CF. Signaling complexes and protein-protein interactions involved in the activation of the Ras and phosphatidylinositol 3-kinase pathways by the c-ret receptor tyrosine kinase. J Biol Chem. 2000; 275(50):39159-66.

14. Colinge J, Cesar-Razquin A, Huber K, Breitwieser FP, Majek P, Superti-Furga G. Building and exploring an integrated human kinase network: global organization and medical entry points. J Proteome. 2014;107:113-27.

15. Bhavanasi D, Badolia R, Manne BK, Janapati S, Dangelmaier CT, Mazharian A, Jin J, Kim S, Zhang X, Chen X, et al. Cross talk between serine/threonine and tyrosine kinases regulates ADP-induced thromboxane generation in platelets. Thromb Haemost. 2015;114(3):558-68.

16. Emkey R, Kahn CR. Cross-talk between phorbol ester-mediated signaling and tyrosine kinase proto-oncogenes. I. Activation of protein kinase $C$ stimulates tyrosine phosphorylation and activation of ErbB2 and ErbB3. J Biol Chem. 1997;272(49):31172-81.

17. Walther TC, Mann M. Mass spectrometry-based proteomics in cell biology. J Cell Biol. 2010;190(4):491-500.

18. Larance M, Lamond Al. Multidimensional proteomics for cell biology. Nat Rev Mol Cell Biol. 2015;16(5):269-80.

19. Neilson KA, Ali NA, Muralidharan S, Mirzaei M, Mariani M, Assadourian G, Lee A, van Sluyter SC, Haynes PA. Less label, more free: approaches in label-free quantitative mass spectrometry. Proteomics. 2011;11(4):535-53.

20. Cox J, Hein MY, Luber CA, Paron I, Nagaraj N, Mann M. Accurate proteomewide label-free quantification by delayed normalization and maximal peptide ratio extraction, termed MaxLFQ. Mol Cell Proteomics. 2014;13(9):2513-26.

21. Kauko $O$, Laajala TD, Jumppanen M, Hintsanen $P$, Suni $V$, Haapaniemi $P$, Corthals G, Aittokallio T, Westermarck J, Imanishi SY. Label-free quantitative phosphoproteomics with novel pairwise abundance normalization reveals synergistic RAS and CIP2A signaling. Sci Rep. 2015;5:13099.

22. Sharma K, D'Souza RC, Tyanova S, Schaab C, Wisniewski JR, Cox J, Mann M. Ultradeep human phosphoproteome reveals a distinct regulatory nature of Tyr and Ser/Thr-based signaling. Cell Rep. 2014;8(5):1583-94.

23. Dazert E, Colombi M, Boldanova T, Moes S, Adametz D, Quagliata L, Roth V, Terracciano L, Heim MH, Jenoe $P$, et al. Quantitative proteomics and phosphoproteomics on serial tumor biopsies from a sorafenib-treated HCC patient. Proc Natl Acad Sci U S A. 2016;113(5):1381-6.

24. Bensimon A, Schmidt A, Ziv Y, Elkon R, Wang SY, Chen DJ, Aebersold R, Shiloh Y. ATM-dependent and -independent dynamics of the nuclear phosphoproteome after DNA damage. Sci Signal. 2010;3(151):rs3.

25. Steger M, Tonelli F, Ito G, Davies P, Trost M, Vetter M, Wachter S, Lorentzen E, Duddy G, Wilson S et al. Phosphoproteomics reveals that Parkinson's disease kinase LRRK2 regulates a subset of Rab GTPases. Elife. 2016;5:e12813.

26. Krishnan N, Lam TT, Fritz A, Rempinski D, O'Loughlin K, Minderman H, Berezney R, Marzluff WF, Thapar R. The prolyl isomerase Pin1 targets stemloop binding protein (SLBP) to dissociate the SLBP-histone mRNA complex linking histone mRNA decay with SLBP ubiquitination. Mol Cell Biol. 2012; 32(21):4306-22.

27. Rich MT, Abbott TB, Chung L, Gulcicek EE, Stone KL, Colangelo CM, Lam TT, Nairn AC, Taylor JR, Torregrossa MM. Phosphoproteomic analysis reveals a novel mechanism of CaMKIlalpha regulation inversely induced by cocaine memory extinction versus reconsolidation. J Neurosci. 2016;36(29):7613-27.

28. Bordner KA, George ED, Carlyle BC, Duque A, Kitchen RR, Lam TT, Colangelo CM, Stone KL, Abbott TB, Mane SM, et al. Functional genomic and proteomic analysis reveals disruption of myelin-related genes and translation in a mouse model of early life neglect. Front Psychiatry. 2011;2:18.

29. Elias JE, Gygi SP. Target-decoy search strategy for mass spectrometry-based proteomics. Methods Mol Biol. 2010;604:55-71.

30. Chou MF, Schwartz D. Biological sequence motif discovery using motif-x. Curr Protoc Bioinformatics. 2011:Chapter 13(Unit 13):15-24.

31. Gnad F, Ren S, Cox J, Olsen JV, Macek B, Oroshi M, Mann M. PHOSIDA (phosphorylation site database): management, structural and evolutionary investigation, and prediction of phosphosites. Genome Biol. 2007;8(11):R250.

32. Kuzmanov U, Guo H, Buchsbaum D, Cosme J, Abbasi C, Isserlin R, Sharma P, Gramolini AO, Emili A. Global phosphoproteomic profiling reveals perturbed signaling in a mouse model of dilated cardiomyopathy. Proc Natl Acad Sci U S A. 2016;113(44):12592-7.

33. Gnad F, Wallin J, Edgar K, Doll S, Arnott D, Robillard L, Kirkpatrick DS, Stokes MP Vijapurkar U, Hatzivassiliou G, et al. Quantitative phosphoproteomic analysis of the PI3K-regulated signaling network. Proteomics. 2016;16(14):1992-7.

34. Possemato AP, Paulo JA, Mulhern D, Guo A, Gygi SP, Beausoleil SA. Multiplexed Phosphoproteomic profiling using titanium dioxide and Immunoaffinity enrichments reveals complementary phosphorylation events. J Proteome Res. 2017;16(4):1506-14.

35. Di Palma S, Zoumaro-Djayoon A, Peng M, Post H, Preisinger C, Munoz J, Heck AJ. Finding the same needles in the haystack? A comparison of phosphotyrosine peptides enriched by immuno-affinity precipitation and metal-based affinity chromatography. J Proteome. 2013;91:331-7.

36. Pan C, Gnad F, Olsen JV, Mann M. Quantitative phosphoproteome analysis of a mouse liver cell line reveals specificity of phosphatase inhibitors. Proteomics. 2008:8(21):4534-46.

37. Courcelles M, Fremin C, Voisin L, Lemieux S, Meloche S, Thibault P. Phosphoproteome dynamics reveal novel ERK1/2 MAP kinase substrates with broad spectrum of functions. Mol Syst Biol. 2013;9:669.

38. Szklarczyk D, Morris JH, Cook H, Kuhn M, Wyder S, Simonovic M, Santos A, Doncheva NT, Roth A, Bork P, et al. The STRING database in 2017: qualitycontrolled protein-protein association networks, made broadly accessible. Nucleic Acids Res. 2017:45(D1):D362-8.

39. Ubersax JA, Ferrell JE Jr. Mechanisms of specificity in protein phosphorylation. Nat Rev Mol Cell Biol. 2007;8(7):530-41.

40. Amanchy R, Periaswamy B, Mathivanan S, Reddy R, Tattikota SG, Pandey A A curated compendium of phosphorylation motifs. Nat Biotechnol. 2007; 25(3):285-6

41. Turk BE. Understanding and exploiting substrate recognition by protein kinases. Curr Opin Chem Biol. 2008;12(1):4-10.

42. Linding $R$, Jensen $L$, Ostheimer GJ, van Vugt MA, Jorgensen C, Miron IM, Diella F, Colwill K, Taylor L, Elder K, et al. Systematic discovery of in vivo phosphorylation networks. Cell. 2007;129(7):1415-26.

43. Van Hoof D, Munoz J, Braam SR, Pinkse MW, Linding R, Heck AJ, Mummery $\mathrm{CL}$, Krijgsveld J. Phosphorylation dynamics during early differentiation of human embryonic stem cells. Cell Stem Cell. 2009;5(2):214-26.

44. Zhou B, Ritt DA, Morrison DK, Der CJ, Cox AD. Protein kinase CK2alpha maintains extracellular signal-regulated kinase (ERK) activity in a CK2alpha kinaseindependent manner to promote resistance to inhibitors of RAF and MEK but not ERK in BRAF mutant melanoma. J Biol Chem. 2016;291(34):17804-15.

45. St-Denis N, Gabriel M, Turowec JP, Gloor GB, Li SS, Gingras AC, Litchfield DW. Systematic investigation of hierarchical phosphorylation by protein kinase CK2. J Proteome. 2015;118:49-62.

46. Zhang X, Maity T, Kashyap MK, Bansal M, Venugopalan A, Singh S, Awasthi S, Marimuthu A, Charles Jacob HK, Belkina N, et al. Quantitative tyrosine Phosphoproteomics of epidermal growth factor receptor (EGFR) tyrosine kinase inhibitor-treated lung adenocarcinoma cells reveals potential novel biomarkers of therapeutic response. Mol Cell Proteomics. 2017;16(5):891-910.

47. Yoshida T, Zhang G, Smith MA, Lopez AS, Bai Y, Li J. Correction: tyrosine Phosphoproteomics identifies both Codrivers and Cotargeting strategies for T790M-related EGFR-TKI resistance in non-small cell lung Cancer. Clin Cancer Res. 2015;21(15):3571

48. Potts MB, Kim HS, Fisher KW, Hu Y, Carrasco YP, Bulut GB, Ou YH, HerreraHerrera ML, Cubillos F, Mendiratta S, et al. Using functional signature ontology (FUSION) to identify mechanisms of action for natural products. Sci Signal. 2013;6(297):ra90.

49. Wang YT, Pan SH, Tsai CF, Kuo TC, Hsu YL, Yen HY, Choong WK, Wu HY, Liao YC, Hong TM, et al. Phosphoproteomics reveals HMGA1, a CK2 substrate, as a drug-resistant target in non-small cell lung Cancer. Sci Rep. 2017;7:44021. 
50. Palvimo J, Linnala-Kankkunen A. Identification of sites on chromosomal protein HMG-I phosphorylated by casein kinase II. FEBS Lett. 1989;257(1):101-4

51. Domizio P, Liesner RJ, Dicks-Mireaux C, Risdon RA. Malignant mesenchymoma associated with a congenital lung cyst in a child: case report and review of the literature. Pediatr Pathol. 1990;10(5):785-97.

52. Schwanbeck R, Gymnopoulos M, Petry I, Piekielko A, Szewczuk Z, Heyduk T, Zechel K, Wisniewski JR. Consecutive steps of phosphorylation affect conformation and DNA binding of the chironomus high mobility group a protein. J Biol Chem. 2001;276(28):26012-21.

53. Adair JE, Kwon Y, Dement GA, Smerdon MJ, Reeves R. Inhibition of nucleotide excision repair by high mobility group protein HMGA1. J Biol Chem. 2005;280(37):32184-92.

54. Maloney SC, Adair JE, Smerdon MJ, Reeves R. Gene-specific nucleotide excision repair is impaired in human cells expressing elevated levels of high mobility group A1 nonhistone proteins. DNA Repair (Amst). 2007:6(9):1371-9.

55. Esposito F, Tornincasa M, Chieffi P, De Martino I, Pierantoni GM, Fusco A. High-mobility group A1 proteins regulate p53-mediated transcription of $\mathrm{BCl}$ 2 gene. Cancer Res. 2010;70(13):5379-88.

56. Lee WK, Son SH, Jin BS, Na JH, Kim SY, Kim KH, Kim EE, Yu YG, Lee HH Structural and functional insights into the regulation mechanism of CK2 by IP6 and the intrinsically disordered protein Nopp140. Proc Natl Acad Sci U S A. 2013;110(48):19360-5.

57. Korolchuk VI, Cozier G, Banting G. Regulation of CK2 activity by phosphatidylinositol phosphates. J Biol Chem. 2005;280(49):40796-801.

58. Solyakov L, Cain K, Tracey BM, Jukes R, Riley AM, Potter BV, Tobin AB. Regulation of casein kinase-2 (CK2) activity by inositol phosphates. J Biol Chem. 2004;279(42):43403-10.

59. Lee YH, Park JW, Bae YS. Regulation of protein kinase CK2 catalytic activity by protein kinase C and phospholipase D2. Biochimie. 2016;121:131-9.

60. Heriche JK, Chambaz EM. Protein kinase CK2alpha is a target for the Abl and Bcr-Abl tyrosine kinases. Oncogene. 1998;17(1):13-8.

61. Donella-Deana A, Cesaro L, Sarno S, Ruzzene M, Brunati AM, Marin O, Vilk G, Doherty-Kirby A, Lajoie G, Litchfield DW, et al. Tyrosine phosphorylation of protein kinase CK2 by Src-related tyrosine kinases correlates with increased catalytic activity. Biochem J. 2003;372(Pt 3):841-9.

62. Donella-Deana A, Cesaro L, Sarno S, Brunati AM, Ruzzene M, Pinna LA. Autocatalytic tyrosine-phosphorylation of protein kinase CK2 alpha and alpha' subunits: implication of Tyr182. Biochem J. 2001;357(Pt 2):563-7.

63. Sarno S, Ghisellini P, Pinna LA. Unique activation mechanism of protein kinase CK2. The N-terminal segment is essential for constitutive activity of the catalytic subunit but not of the holoenzyme. J Biol Chem. 2002;277(25): 22509-14.

64. Watanabe N, Broome M, Hunter T. Regulation of the human WEE1Hu CDK tyrosine 15-kinase during the cell cycle. EMBO J. 1995;14(9):1878-91.

65. Fattaey A, Booher RN. Myt1: a Wee1-type kinase that phosphorylates Cdc2 on residue Thr14. Prog Cell Cycle Res. 1997;3:233-40.

66. Uckun FM, Tuel-Ahlgren L, Waddick KG, Jun X, Jin J, Myers DE, Rowley RB, Burkhardt AL, Bolen JB. Physical and functional interactions between Lyn and p34cdc2 kinases in irradiated human B-cell precursors. J Biol Chem. 1996;271(11):6389-97.

67. Deutsch EW, Csordas A, Sun Z, Jarnuczak A, Perez-Riverol Y, Ternent T, Campbell DS, Bernal-Llinares M, Okuda S, Kawano S, et al. The ProteomeXchange consortium in 2017: supporting the cultural change in proteomics public data deposition. Nucleic Acids Res. 2017;45(D1):D1100-6.

68. Vizcaino JA, Csordas A, del-Toro N, Dianes JA, Griss J, Lavidas I, Mayer G, Perez-Riverol Y, Reisinger F, Ternent T, et al. 2016 update of the PRIDE database and its related tools. Nucleic Acids Res. 2016;44(D1):D447-56.

69. Eid S, Turk S, Volkamer A, Rippmann F, Fulle S. KinMap: a web-based tool for interactive navigation through human kinome data. BMC Bioinformatics. 2017;18(1):16.

Ready to submit your research? Choose BMC and benefit from:

- fast, convenient online submission

- thorough peer review by experienced researchers in your field

- rapid publication on acceptance

- support for research data, including large and complex data types

- gold Open Access which fosters wider collaboration and increased citations

- maximum visibility for your research: over $100 \mathrm{M}$ website views per year

At BMC, research is always in progress.

Learn more biomedcentral.com/submissions 